\title{
Hernia de Garengeot y revisión de las variantes de hernias encarceladas
}

\author{
Garengeot's hernia and review of variants of incarcerated hernias
}

\author{
Ana Romero $^{1} \mathbb{D}$, Diana Robayo ${ }^{2} \mathbb{D}$, Eric $\operatorname{Vinck}^{3} \mathbb{D}$, Alejandra Triviño ${ }^{2} \mathbb{D}$, Stefanía Sacipa ${ }^{4} \mathbb{D}$, \\ Efraín Gómez ${ }^{1} \mathbb{D}$
}

1. Médico, especialista en Cirugía general, Hospital Simón Bolívar, Bogotá D.C., Colombia.

2. Médico, servicio de Cirugía general, Hospital Simón Bolívar, Bogotá D.C., Colombia.

3. Médico, fellow de Cirugía cardiovascular, Clínica Cardio-VID, Medellín, Colombia.

4. Médico, servicio de Cirugía general, Hospital de Zipaquirá, Zipaquirá, Colombia.

\section{Resumen}

Introducción. La hernia de Garengeot se caracteriza por contener el apéndice cecal dentro del saco femoral, y forma parte de una variedad de hernias que reciben el epónimo de acuerdo con su localización anatómica. Entre ellas se encuentra la hernia de Richter, la hernia de Amyand, la hernia de Littré y la hernia de Spiegel. Se presenta una revisión de los abordajes laparoscópicos para estas diversas variantes.

Caso clínico. Paciente femenina de 82 años de edad quien consultó al servicio de urgencias por dolor inguinal derecho de ocho días de evolución, asociado a clínica de obstrucción intestinal. Se hizo diagnóstico de hernia inguinal encarcelada y se llevó a cirugía encontrando una hernia de Garengeot.

Discusión. Además de los tipos de hernia tradicionalmente conocidos, existen variantes inusuales de hernias de la pared abdominal, que deben ser sospechadas y tenidas en cuenta como diagnóstico diferencial, lo que permitirá realizar su tratamiento de forma oportuna disminuyendo el riesgo de que ocurra una perforación intestinal.

Conclusiones. Las variantes de hernia inguinal o de localización inusual, son susceptibles de tratamiento quirúrgico mediante abordajes laparoscópicos con adecuados resultados.

Palabras clave: hernia; Garengeot; apendicitis; obstrucción; laparoscopia.

\footnotetext{
Abstract

Introduction. Garengeot's hernia is characterized by containing the cecal appendix within the femoral sac, and is part of a variety of hernias that receive their eponymous according to their anatomical location. These include Richter's hernia, Amyand's hernia, Littré's hernia, and Spiegel's hernia. We present a review of the laparoscopic approaches for these variants.

Fecha de recibido: 12/02/2021 - Fecha de aceptación: 29/03/2021 - Publicación en línea: 09/11/2021

Correspondencia: Diana Robayo, Calle 165 \# 7 - 06, Bogotá, D.C., Colombia. Teléfono: +57 3112491157

Dirección electrónica: dxr.9508@gmail.com

Citar como: Romero A, Robayo D, Vinck E, Triviño A, Sacipa S, Gómez E. Hernia de Garengeot y revisión de las variantes de hernias encarceladas. Rev Colomb Cir. 2022;37:122-8. https://doi.org/10.30944/20117582.893

Este es un artículo de acceso abierto bajo una Licencia Creative Commons - BY-NC-ND https://creativecommons.org/licenses/by-nc-nd/4.0/deed.es
} 
Clinical case. An 82-year-old female patient consulted to the emergency department for right groin pain of eight days of evolution, associated with symptoms of intestinal obstruction. With a diagnosis of incarcerated inguinal hernia, she underwent surgery finding a Garengeot's hernia.

Discussion. In addition to the traditionally known types of hernia, there are unusual variants of hernias of the abdominal wall, which must be suspected and taken into account as a differential diagnosis, which will allow treatment to be carried out in a timely manner, reducing the risk of intestinal perforation.

Conclusions. Variants of inguinal hernia or unusual location are susceptible to surgical treatment by laparoscopic approaches with adequate results.

Keywords: hernia; Garengeot; appendicitis; obstruction; laparoscopy.

\section{Introducción}

Se conoce como hernia de Garengeot a la presencia del apéndice cecal dentro del saco herniario de una hernia femoral. Fue descrita por Jacques Croissant De Garengeot en 1731, y es una variedad clínica poco frecuente, que conlleva dificultades diagnósticas para el cirujano general ${ }^{1}$. Representa el 0,9\% de las hernias, y corresponde a tan solo el 0,13\% - 0,8\% de los casos de apendicitis aguda ${ }^{1,2}$. Se considera que ocurre debido a una variante anatómica de la implantación del apéndice o a un ciego de mayor tamaño y movilidad que favorecen la encarcelación del apéndice en una hernia femoral ${ }^{3}$.

Las hernias de la pared abdominal ocurren especialmente en la región inguinocrural y contienen epiplón o intestino delgado, pero también puede estar presente en ellas: un divertículo de Meckel, situación conocida como hernia de Littré, un segmento de pared intestinal como en la de Richter, o el apéndice cecal, como en el caso de las hernias de Amyand y Garengeot ${ }^{3}$. Se presenta el caso de una paciente con diagnóstico de hernia femoral encarcelada, que fue llevada a cirugía de urgencia por obstrucción intestinal, con hallazgo intraoperatorio de hernia de Garengeot. Se describen además la técnica laparoscópica para el tratamiento de estas variantes infrecuentes.

\section{Presentación del caso}

Paciente femenina de 82 años de edad, quien ingresó al servicio de urgencias por dolor inguinal derecho de ocho días de evolución, asociado a dos episodios eméticos. Como único antecedente de importancia refirió hipertensión arterial tratada con losartán. Al examen físico con tensión arterial de 124/68 mmHg, frecuencia cardiaca de 72 latidos por minuto, frecuencia respiratoria de 18 respiraciones por minuto, saturación de oxígeno de $96 \%$, afebril, sin signos de respuesta inflamatoria sistémica. Como único hallazgo positivo se encontró una hernia inguinal derecha encarcelada, sin signos de irritación peritoneal. Hemograma con 8760 leucocitos, neutrófilos $82,3 \%$, linfocitos $11,4 \%$, hemoglobina de $14,2 \mathrm{~g} / \mathrm{dL}$, hematocrito de $21 \%$, VCM 94,5 fl, HCM 32,8 p/c, plaquetas $363,000 \mathrm{mcL}$, creatinina $0,51 \mathrm{mg} / \mathrm{dL}$, nitrógeno ureico 13,6 mg/dL y PCR ligeramente elevada en $54 \mathrm{mg} / \mathrm{L}$.

Con diagnóstico de hernia inguinal derecha encarcelada y obstrucción intestinal la paciente fue llevada a cirugía mediante un abordaje abierto con incisión derecha tipo Nyhus, disección por planos hasta el espacio preperitoneal de Bogros, disección digital hasta identificar la hernia estrangulada. Se realizó apertura de ligamento lacunar logrando liberación del anillo herniario y reducción de la hernia femoral, después de seccionar el saco herniario se encontró un segmento de epiplón, cinco $\mathrm{cm}$ de íleon distal que al reducirlo recuperó su peristaltismo y perfusión, y el apéndice cecal con hiperemia en la serosa (Figura 1). Se realizó apendicectomía, omentectomía parcial y resección y ligadura alta del saco con Vicryl ${ }^{\circledR}$. El defecto herniario fue corregido con una malla de polipropileno que se fijó al ligamento de Cooper. El procedimiento fue realizado sin complicaciones y la paciente evolucionó adecuadamente siendo dada de alta. 


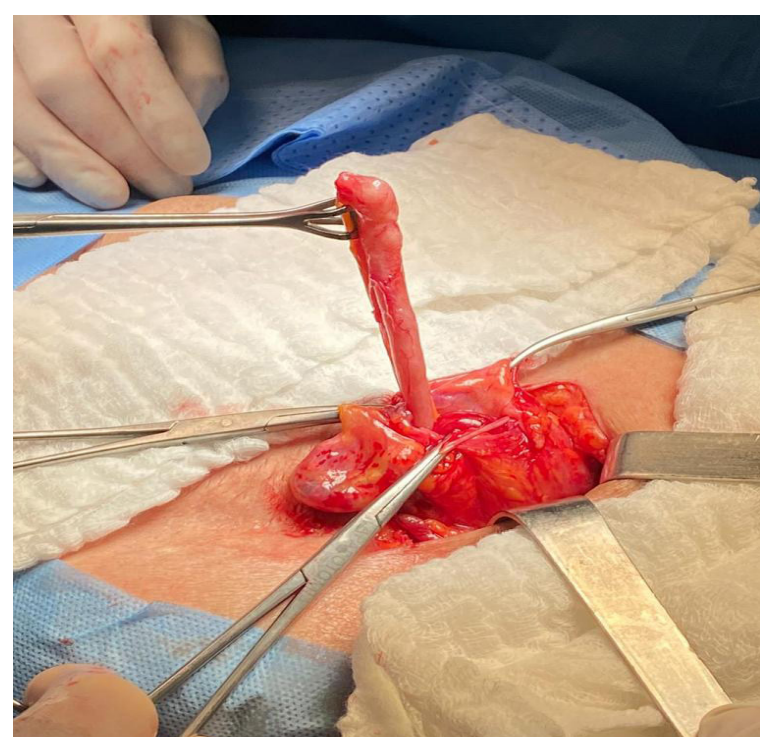

Figura 1. Apéndice cecal con hiperemia, contenida en el saco herniario femoral derecho.

\section{Revisión del tema}

\section{Hernia de Amyand}

Corresponde a una hernia inguinal que dentro de su saco contiene el apéndice cecal con o sin signos de inflamación o perforación. Fue descrita en el año 1735 por el cirujano francés Claudius Amyand en el St. George's Hospital de Londres, quien además realizó la primera apendicectomía exitosa en un niño de 11 años de edad. ${ }^{4}$.

Se caracteriza por el hallazgo de una hernia encarcelada con dolor tipo cólico y signos de defensa abdominal, el diagnóstico generalmente se realiza de forma intraoperatoria ${ }^{5}$. En caso de encontrar un apéndice sano se recomienda el manejo con antibióticos, y solo realizar apendicectomía en casos de inflamación o perforación apendicular. El uso de mallas protésicas generalmente se difiere por el alto riesgo de infección o fístula del muñón apendicular. Si se realiza la apendicectomía y la corrección de la hernia, se recomienda hacerlo por la misma incisión ${ }^{6}$.

Losanoff y Basson describieron las características de la reparación herniaria basados en cuatro estados del apéndice cecal: apéndice cecal normal, apéndice cecal con algún grado de apendicitis aguda, apéndice cecal con apendicitis aguda más sepsis abdominal o apendicitis aguda perforada, y apéndice cecal con apendicitis aguda y otras enfermedades de origen abdominal no asociadas ${ }^{7}$; posteriormente Rikki et al. modificaron esta clasificación ampliando el manejo quirúrgico según el estado del apéndice incluido dentro de una hernia incisional ${ }^{8}$.

\section{Hernia de Richter}

La hernia de Richter es la protrusión del borde antimesentérico de un asa intestinal a través de un defecto de la pared abdominal; el segmento más comúnmente comprometido es el íleon terminal. Este tipo de hernia fue descrita por primera vez en el año 1598 por el médico alemán Fabricius Hildamus, quien durante un largo periodo de la historia ha sido mencionado por diferentes cirujanos, sin embargo, la hernia recibe este epónimo por el doctor Richter quien fue el primero en publicarla en $1785^{9}$.

La obstrucción en este tipo de hernia es muy rara, debido a que no se compromete la totalidad de la luz intestinal, pero a pesar de esto, el riesgo de isquemia o perforación del segmento intestinal es muy alto dado la pobre vascularización que presenta el borde antimesentérico. Se puede identificar en cualquier localización, pero ocurre más comúnmente en las hernias femorales e inguinales ${ }^{10}$. Dada la presentación y el curso clínico de esta hernia, el diagnóstico suele ser tardío, lo que se relaciona con un mayor riesgo de complicaciones y morbimortalidad. El manejo es quirúrgico y la necesidad de resección del segmento intestinal depende de la viabilidad del mismo ${ }^{10}$.

\section{Hernia de Littré}

Se caracteriza por la protrusión de un divertículo de Meckel a través de un defecto herniario en la pared abdominal. Esta patología fue descrita por primera vez por el doctor Fabricius Hildamus en 1598, sin embargo, solo en 1809 se hace la descripción macroscópica y el origen embrionario del divertículo por el anatomista y embriólogo alemán Johann Friedrich Meckel, por quien recibe el nombre de divertículo de Meckel. En 1700 el cirujano y anatomista francés Alexis Littré publi- 
ca la presencia del divertículo dentro de un saco herniario ${ }^{11}$.

El divertículo de Meckel es una anomalía gastrointestinal congénita común, que corresponde a la persistencia del conducto onfalomesentérico, el cual usualmente se oblitera después a la quinta semana de gestación. Tiene una incidencia del $0,3-3 \%$ de la población, siendo 3 - 4 veces más prevalente en hombres, se localiza en los $60 \mathrm{~cm}$ del íleon terminal y mide entre 4 a $6 \mathrm{~cm}$ de longitud y $2 \mathrm{~cm}$ de diámetro. Solo en el $10 \%$ de los casos es sintomático ${ }^{12}$, manifestándose mediante sangrado o infección, que puede simular una apendicitis aguda ${ }^{13}$.

La hernia de Littré es poco frecuente y se presenta en un 1 - $2 \%$ de los casos de las personas que presentan un divertículo de Meckel ${ }^{12,13}$. La ubicación anatómica varía, encontrándose más frecuentemente en la hernia inguinal derecha en un $50 \%$ de los casos, seguida de la femoral y la umbilical en 20 - $30 \%$, sin embargo, también se han encontrado en hernias ventrales y obturatrices en un $10 \%{ }^{12,14}$. El tratamiento es quirúrgico ya sea por vía abierta o laparoscopia, además de electivo o urgente, y consiste en la resección del divertículo mediante una cuña si es un hallazgo incidental, o segmentaria si es una cirugía de urgencia, luego se realiza la anastomosis del íleon y la reparación quirúrgica de la hernia, la cual se puede hacer con malla, dependiendo de la contaminación ${ }^{13,15}$.

\section{Hernia de Garengeot}

La hernia de Garengeot es la migración del apéndice al saco herniario de una hernia femoral. Fue descrita por primera vez por el cirujano francés René Jacques Croissant De Garengeot en 1731 quien identificó una apendicitis aguda contenida en una hernia femoral encarcelada ${ }^{3}$, sin embargo, fue en 1785 cuando Hevin realizó la primera apendicectomía de una hernia femoral ${ }^{1}$. Las hernias femorales son más frecuentes en mujeres que en hombres, en relación 2:1, con predisposición en mujeres posmenopáusicas. La prevalencia en las mujeres se da por cambios corporales del em- barazo y factores como presión intraabdominal, tabaquismo, edad avanzada y defectos de colágeno ${ }^{3}$.

El hallazgo del apéndice dentro de una hernia femoral se presenta en el 0,9\% de los casos, sin embargo, la apendicitis dentro del canal femoral solo corresponde al 0,13\% - 0,8 \% de todos los casos de apendicitis aguda ${ }^{16}$. Debido al carácter rígido y estrecho del canal inguinal, se condiciona un riesgo de encarcelamiento de hasta el $14-56 \%{ }^{1}$, presentando una clínica de protuberancia inguinal irreductible, dolorosa, con signos inflamatorios, y abdomen agudo.

El manejo es quirúrgico mediante apendicectomía por vía abierta con posterior corrección de la hernia o apendicectomía a través del saco herniario. Se recomienda el uso de malla en caso de no existir perforación o absceso, ya que de lo contrario se aumenta el riesgo de infección o recurrencia de la hernia ${ }^{3,16}$.

\section{Hernia de Spiegel}

La protrusión de grasa preperitoneal a través de un defecto herniario congénito o adquirido de la fascia de Spiegel, recibe este epónimo desde 1764 cuando Klinkosch describió la hernia ventral lateral espontánea situada en la línea semilunar de Spiegel, identificada por primera vez en 1645 por el anatomista francés Adrian Van Spiegel. También es conocida como hernia ventral lateral espontánea, de la línea semilunar, intersticial o interparietal ${ }^{17-18}$.

Este tipo de hernia es muy rara, corresponde a menos del $2 \%$ de todas las hernias, el $90 \%$ se encuentran en el "cinturón de Spiegel", y en los casos descritos se han encontrado factores predisponentes como tos o neumopatías crónicas, obesidad, diálisis peritoneal, multiparidad, estreñimiento, ascitis, y cirugías previas, entre otras ${ }^{19-20}$. Se puede asociar a otras hernias de la pared abdominal hasta en un $50 \%$ de los casos ${ }^{17}$. El tratamiento es quirúrgico por vía abierta o laparoscopia, y su recidiva es del $4 \%$ en el abordaje laparoscópico, mientras que por vía abierta es casi de $0 \%{ }^{21}$. 


\section{Abordaje laparoscópico}

Generalmente el diagnóstico de este tipo de hernias se realiza de forma intraoperatoria y la cirugía se realiza como una urgencia, aun así, el abordaje laparoscópico es posibles en ciertos casos. En la literatura existen algunos reportes de hernias de Amyand corregidas por vía laparoscópica, pero aún no se puede recomendar este abordaje mínimamente invasivo con la técnica estándar. En 2016, Shaknovsky et al. publicaron el caso de un paciente con hernia de Amyand tratado exitosamente mediante cirugía robótica, ofreciendo una posibilidad quirúrgica mínimamente invasiva ${ }^{22}$.

En cuanto a las hernias de Spiegel, en 2019, Machado y cols. reportaron un caso exitoso de reparación por medio de laparoscopia en la ciudad de Medellín ${ }^{23}$. Dada la poca frecuencia y el subdiagnóstico de la hernia de Spiegel se presenta una limitación en el estudio de las técnicas quirúrgicas para definir su manejo ideal. Después de la técnica abierta, el abordaje más recomendado es el laparoscópico con técnica IPOM (intraperitoneal onlay mesh), que es más fácil, ocupa menor tiempo quirúrgico y no requiere crear un colgajo peritoneal, que presenta como desventajas la entrada a la cavidad peritoneal y un mayor riesgo de inflamación y adherencias ${ }^{23,24}$.

En segundo lugar, se encuentra la técnica TAPP (transabdominal preperitoneal), un procedimiento con disección preperitoneal, que permite visualizar el contenido del saco herniario y cerrar el defecto, con la ventaja de que la malla queda preperitoneal sin contacto con las asas intestinales ${ }^{23}$. Finalmente está disponible la técnica TEP (totalmente extraperitoneal) que presenta mayor dificultad respecto a las anteriores, requiere mayor experiencia del cirujano y no permite la adecuada exploración del contenido del saco herniario, por lo que no se recomienda su uso en cirugía de urgencia ${ }^{23-25}$.

Las hernias de Richter pueden localizarse en diferentes sitios como el orificio inguinal, crural, femoral, umbilical, obturador e incluso en los de los puertos de laparoscopia. Su abordaje quirúrgico depende de la localización, de la clínica del paciente y de la experiencia del cirujano. El abordaje laparoscópico, tanto preperitoneal como intraperitoneal, presentan resultados adecuados con menor dolor posoperatorio, menor número de complicaciones pulmonares y de días de estancia hospitalaria ${ }^{26}$.

En la hernia de Garengeot, además de la vía abierta con apendicectomía a través del saco herniario, se describen diversos abordajes laparoscópicos, que dependen de las comorbilidades, condiciones del paciente, y de la pericia del cirujano.

Mediante esta técnica de mínima invasión se realiza la apendicectomía y la corrección de la hernia con técnica TAPP o TEP ${ }^{27-29}$. También se ha descrito la reparación del defecto herniario en un segundo tiempo quirúrgico ${ }^{27}$. Ramsingh et al. describieron el primer abordaje combinado en hernia de Garengeot con herniorrafia por vía inguinal y apendicectomía vía laparoscópica ${ }^{30}$. En pacientes con sospecha de proceso infeccioso que requieren lavado quirúrgico local se recomiendan abordajes combinados ${ }^{27,28,30}$.

\section{Conclusión}

En pacientes con hernia de Garengeot, una vez identificado el apéndice cecal dentro del saco herniario, se debe realizar apendicectomía, y reparación de la hernia con malla dependiendo de un campo quirúrgico no contaminado. La intervención quirúrgica no se debe retardar en espera de imágenes diagnósticos, ya que estas variantes pueden llegar a presentar apendicitis aguda y perforación intestinal por el componente obstructivo.

Además de las formas usuales de hernia de pared abdominal, existen unas variantes de baja incidencia que deben permanecer en la mente del cirujano y ser tenidas en cuenta como diagnóstico diferencial. A pesar de que generalmente requieren un tratamiento quirúrgico de urgencias, el abordaje laparoscópico es posible en ciertos casos y depende del estado del paciente y de la experiencia del cirujano.

\section{Cumplimiento de normas éticas}

Consentimiento informado: La paciente dio su consentimiento informado para la publicación de este caso. 
Conflicto de interés: Ninguno declarado por los autores.

Fuente de financiación: Financiado con recursos propios de los autores.

\section{Contribución de los autores}

Concepción y diseño del estudio: Ana Romero, Diana Robayo, Eric Vinck, Alejandra Triviño, Stefanía Sacipa, Efraín Gómez.

Adquisición de datos: Ana Romero, Diana Robayo, Eric Vinck, Alejandra Triviño, Stefanía Sacipa, Efraín Gómez.

Análisis e interpretación de datos: Ana Romero, Diana Robayo, Eric Vinck, Alejandra Triviño, Stefanía Sacipa, Efraín Gómez.

Redacción del manuscrito: Ana Romero, Diana Robayo, Eric Vinck, Alejandra Triviño, Stefanía Sacipa, Efraín Gómez.

Revisión crítica: Ana Romero, Diana Robayo, Eric Vinck, Alejandra Triviño, Stefanía Sacipa, Efraín Gómez.

\section{Referencias}

1. Aznar-Jean A, Acevedo-Estrada RI. Reporte de caso de hernia de Garengeot en un centro de cirugía ambulatoria. Rev Esp Med Quir. 2015;20:210-4.

2. Hernández A, León AM, Murillo A. Hernia de Garengeot: Reporte de caso y revisión de la literatura. Cir Gen. 2012;34:78-82.

3. Santiesteban-Pupo WE, Borges-Sandrino RS, Ramón-Musibay E. Hernia de Littré. Rev Cubana Med Milit. 2018;47:1-7.

4. Singhal S, Singhal A, Negi SS, Tugnait R, Arora PK, Tiwari $B$, et al. Amyand's hernia: rare presentation of a common ailment. Case reports in gastrointestinal medicine. 2015;1:1-7. https://doi.org/10.1155/2015/629127

5. Fonseca-Sosa FK, Casado-Méndez PR, Santos-Fonseca RS, Núñez-Siré RA, Rey-Vallés YS, Quesada-Martínez E. Hernia de Amyand. Reporte de caso. Rev Med Electrón. 2017;39:330-7.

6. López-La F. Reporte de un paciente con hernia de Amyand. CCM. 2015;19:153-9.

7. Sardiñas-Ponce R, Hernández-Torres L, Pinza-Jojoa J. Hernia de Amyand. Presentación de un caso. Medisur. 2015;13:677-80.

8. Villarreal R, Luna-Jaspe C, Cabrera LF, Vinck E. Hernia de Amyand encarcelada, revisión de la literatura y reporte de un caso en una institución de tercer nivel en Bogotá. Rev Colomb Cir. 2016;31:283-8.

9. Benítez G, Nakhal E, Scorzza R, Saade R, García E. Hernia intra abdominal de Richter. Revista de la Facultad de Medicina. 2004;27:15-18.
10. Alarcón del Agua I, Jiménez-Rodríguez R, Suárez-Artacho G, García B. Hernia de Richter como causa de obstrucción intestinal de difícil diagnóstico. Rev Esp Enferm Dig. 2010;102:611-2. https://doi.org/10.4321/S1130-01082010001000012

11. Mustelier-Sánchez RM, González de la Fe R. Hernia de Littré estrangulada. Presentación de un caso. Multimed. 2019;23:1112-23.

12. Velásquez-Bueso AE, Sánchez-Sierra LE, Villeda-Rodríguez SD, Martínez-Quiroz RA. Simultaneous presentation of a right Littre's hernia and a left Amyand's hernia in a school-aged patient. Case Reports in Surgery. 2019;10:4217329. https://doi.org/10.1155/2019/4217329

13. Oliu-Lambert H, De la Cruz-Castillo NA, Pineda-Chacón J. Hernia de Littré estrangulada en un adulto. Medisan. 2017;21:3367-71.

14. Luengas JP, Sacristán PA, Sáenz-Rey K, Sanabria-Esguerra I. Hernia de Littré estrangulada. A propósito de un caso. Revista Med. 2016;24:81-7. https://doi.org/10.18359/rmed.2644

15. Talini C, Oliveira LO, Araújo AC, Netto FA, Westphalen AP. De Garengeot hernia: case report and review. International Journal of Surgery Case Reports. 2015;8:35-7. https://doi.org/10.1016/j.ijscr.2014.12.042

16. Caraballoso-García VJ, Cabrera-Reyes J, Alonso-Domínguez N, Santana-González-Chávez A, Orea-Cordero I. Hernia de Garengeot. A propósito de un caso. Rev Med Electrón. 2018;40:488-94.

17. Fonseca-Sosa FK, Rey-Vallés YS. Hernia de Spiegel: diagnóstico dudoso. Multimed. 2019;23:1395-402.

18. Ruiz de la Hermosa A, Amunategui-Prats I, Machado-Liendo P, Nevarez-Noboa F, Muñoz-Calero A. Hernias de Spiegel: Nuestra experiencia y revisión de la literatura. Rev Esp Enferm Dig. 2010;102:583-6. https://doi.org/10.4321/S1130-01082010001000003

19. Ye Z, Wang MJ, Bai LF, Zhuang HX, Zhuang W. Spigelian hernia in the right upper abdominal wall: a case report. BMC surgery. 2018;18:1-3. https://doi.org/10.1186/s12893-018-0449-5

20. García-Mendez LR, Rodríguez-García L. Hernia de Spiegel. A propósito de un caso. Rev Med Electrón. 2019;41:748-55.

21. Cruz-Rodríguez J, Monteagudo-Ramírez J, Ramos-Rodríguez J. Hernia de Spiegel: presentación de un caso. Gac Méd Espirit. 2019;21:121-7.

22. Shaknovsky T, Sabido F, Shaikh D, Rosen P. Robotic repair of Amyand's hernia: A case report. J Case Rep Images Surg. 2016;2:92-6.

23. Machado F, Calle C, López F. Hernia de Spiegel como causa de obstrucción intestinal: abordaje laparoscópico. Rev Hispanoam Hernia. 2019;7:72-5. http://dx.doi.org/10.20960/rhh.184 
24. Dzib-Calan E, Ortiz-Reyes S, Morales-Pérez J, Núñez-Vidales R, Rodarte-Cajica G, Leal-Mérida G. Reparación laparoscópica de hernia de Spiegel con colocación de malla compuesta intraperitoneal y sistema de fijación articulado. Reporte de dos casos. Cir Cir. 2019;87:353-7. https://doi.org/10.24875/CIRU.18000484

25. Zacharoulis D, Sioka E. Laparoscopic Spigelian hernia repair: intraperitoneal onlay mesh-plus technique video presentation. J Laparoendosc Adv Surg Tech A. 2018;28:201-3. https://doi:10.1089/lap.2017.0009

26. Allamand T. J, Schönffeldt G. K, Campero M. J, Santibáñez P. C, Rojas F. N. Obstrucción intestinal por hernia obturatriz: Serie de casos. Rev Cir. 2019;71:173-7. https://doi.org/10.4067/s2452-45492019000200173

27. Briceño E, Jara R. Fascitis necrotizante de la pared abdominal como presentación infrecuente de una hernia de Garengeot: Caso clínico. Rev méd Chile. $2018 ; 146: 660-4$

https://doi.org/10.4067/s0034-98872018000500660

28. Al-Subaie S, Mustafa H, Al-Sharqawi N, Al-Haddad M, Othman F. A case of de Garengeot hernia: the feasibility of laparoscopic transabdominal preperitoneal hernia repair. Int J Surg Case Rep. 2015;16:73-6.

29. Beysens M, Haeck Y L, Vindevoghel K. Laparoscopic Appendectomy Combined with TEP for de Garengeot Hernia: Case Report. Acta Chir Belg. 2013;113:468-70.

30. Ramsingh J, Ali A, Cameron C, Al-Ani A, Hodnett R, Chorushyj C. De Garengeot's hernia: diagnosis and surgical management of a rare type of femoral hernia. Journal of Surgical Case Reports. 2014;2:1-3.

https://doi.org/10.1093/jscr/rju008 